\title{
32. METEOR MASS DISTRIBUTION FROM RADAR OBSERVATIONS
}

\author{
B. A. McIntosh \\ (National Research Council, Ottawa, Canada)
}

\begin{abstract}
A BSTRACT
There are many problems in deducing the meteor mass distribution from radar echo observations. Some of these are reviewed in this paper, particular emphasis being placed on the effects of electron attachment and of equipment parameters on the statistics of long-duration echoes.

The ability of radar to detect meteors from the very brightest down to 15 th magnitude makes it seem attractive as a method of examining the size distribution of the incoming particles over a wide range. Some of the problems involved in determining the meteor mass distribution from radar observations are discussed in this paper. Particular emphasis is placed on the effects of electron attachment and of equipment parameters on the statistics of long-duration echoes.

In theory the electron line density, $q$, in a meteor trail is linearly related to the mass, $m$, of the meteoroid producing it. If the number of meteoroids, $N_{\mathrm{m}}$, having mass $m$ and greater is expressible as

$$
N_{\mathrm{m}} \propto m^{-(s-1)}
$$

then the relative distribution of trails having line densities $q$ and greater will be

$$
N_{q} \propto q^{-(s-1)}
$$

Hence determination of this relation from radar observations will yield the mass exponent $s$.

The reflection properties of trails are intrinsically different according to whether the electron line density $q$ is less than (underdense), or greater than (overdense) about $10^{14} \mathrm{el} / \mathrm{m}$. The measured parameter which is the more suitable for the determination of $q$ is different in the two regimes.
\end{abstract}

\section{Underdense Echoes}

For underdense echoes maximum received power is $P_{\mathrm{R}} \propto q^{2}$ and the echo decays as $\exp \left(-32 \pi^{2} D t / \lambda^{2}\right)$. With an equipment capable of measuring received echo power over a fairly wide range, the mass exponent $s$ may be obtained from observations 
yielding the relation

$$
\log N_{\mathrm{p}}=\text { constant }-\frac{s-1}{2} \log P_{\mathbf{R}}
$$

A typical set of such measurements is shown in Figure 1. It is evident that a linear relation between $\log N$ and $\log P_{\mathrm{R}}$ is valid over most of these data. There is a slight

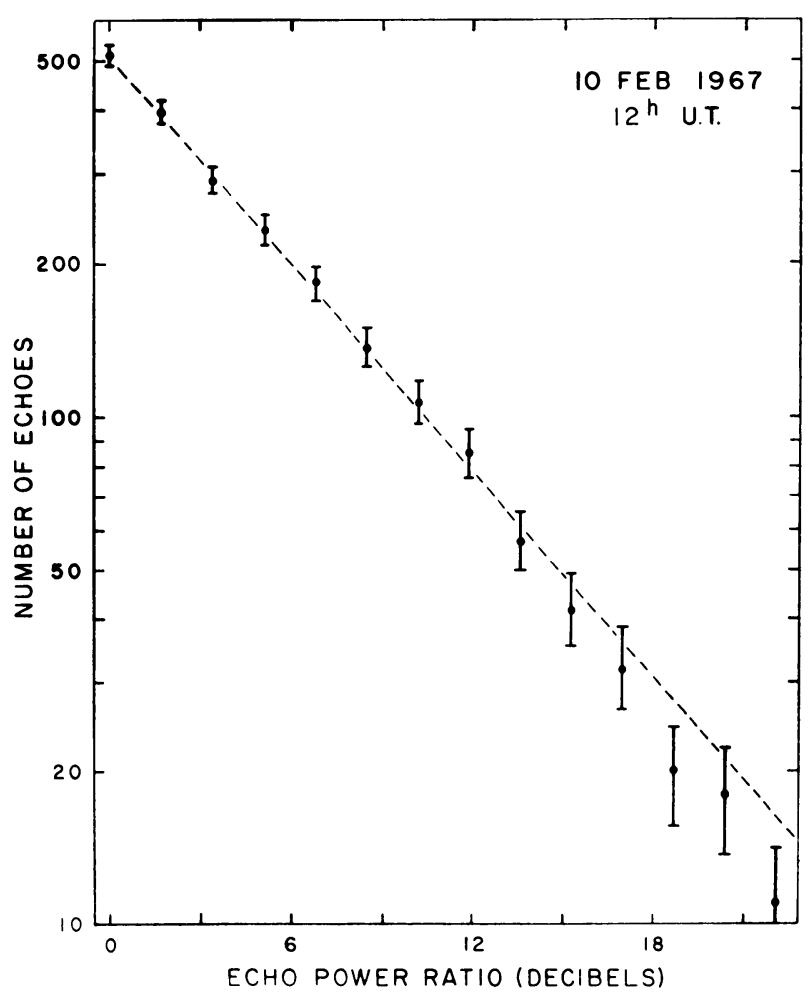

FIG. 1. Plot of the number of echoes from underdense trails with received power greater than or equal to the abscissal values.

tendency here, and in other cases, for the slope to increase toward the stronger echoes. This is believed to be due to the fact that the broad transition region between the underdense and overdense cases is being approached. Problems in the interpretation of these results are dealt with in more detail in the paper by Simek and McIntosh (1968).

A problem which has not been resolved is to what extent the so-called radar height ceiling affects these measurements. Greenhow and Hall (1960) believed that $s$ values derived in this way were too low because the height ceiling reduced the number of small meteoroids that could be observed. From observations of the 1966 Leonid meteor shower we have deduced an $s$ value of $2 \cdot 2 \pm 0 \cdot 2$ from overdense trails. From 
amplitude measurements of underdense trails a tentative value $s=1 \cdot 7$ has been deduced. It cannot be shown definitely that this is not a real change in the mass distribution. However, the evidence indicates that the concentration of Leonid meteors was of recent origin so that there has not been sufficient time for the small particles to be removed. Further, because the height-ceiling cut-off is most severe for high-velocity meteors, it seems possible that the observed dearth of faint Leonids may be due to this.

\section{Overdense Echoes}

The amplitude method is not very sensitive for overdense echoes since $P_{\mathrm{R}} \propto q^{1 / 2}$. Furthermore, echoes may be distorted, having fading and fluctuating amplitude. The usual parameter measured is echo duration $\tau$ since more nearly, $\tau \propto q$. Duration is controlled by atmospheric parameters as well as line density, and depends on height of maximum ionization which is both mass and velocity dependent. The slower and/or more massive particles penetrate to lower heights. At great heights, diffusion is the controlling factor and the number of echoes having duration $\tau$ and greater may be shown to be

$$
N_{\tau} \propto \tau^{-3(s-1) / 4} .
$$

Lower in the atmosphere attachment of electrons to neutral molecules becomes the dominant decay process and

$$
N_{T} \propto T^{-9(s-1) / 2} .
$$

Figure 2 shows the log number vs. log duration relation in completely normalized form. It is important to note from this curve that the transition from asymptotic slope -1 to asymptotic slope -6 is long. This is emphazised by Figure 3 which shows that the slope is changing over a range of duration of nearly 100 times. Thus it is dangerous to infer values of $s$ from observations comprising only a short range of echo durations, particularly if the value of the normalizing duration constant $\tau_{0}$ is not known. It has been shown by McIntosh (1966) that $\tau_{0}$ is given by

$$
\tau_{0} \simeq 0.53 \lambda^{2 / 5} v^{2} B_{93}^{-4 / 5}
$$

where $\lambda$ is radar wavelength in metres, $v$ is meteor velocity in units of $40 \mathrm{~km} / \mathrm{sec}$, and $B_{93}$ is the value of attachment rate at a height of $93 \mathrm{~km}$.

Typical values for $\lambda=9.2 \mathrm{~m}, B_{93}=0.02 \mathrm{sec}^{-1}$ are

$$
\begin{aligned}
& V=20 \mathrm{~km} / \mathrm{sec}, \quad \tau_{0}=7 \mathrm{sec}, \\
& V=70 \mathrm{~km} / \mathrm{sec}, \quad \tau_{0}=90 \mathrm{sec} .
\end{aligned}
$$

It is possible to calculate approximately the absolute echo duration to be expected from a meteoroid of given mass. Some results obtained by McIntosh (1967) are shown in Figure 4. The drastic reduction in echo duration due to attachment is apparent. Maximum echo durations of $200-300 \mathrm{sec}$ are typical of values obtained 


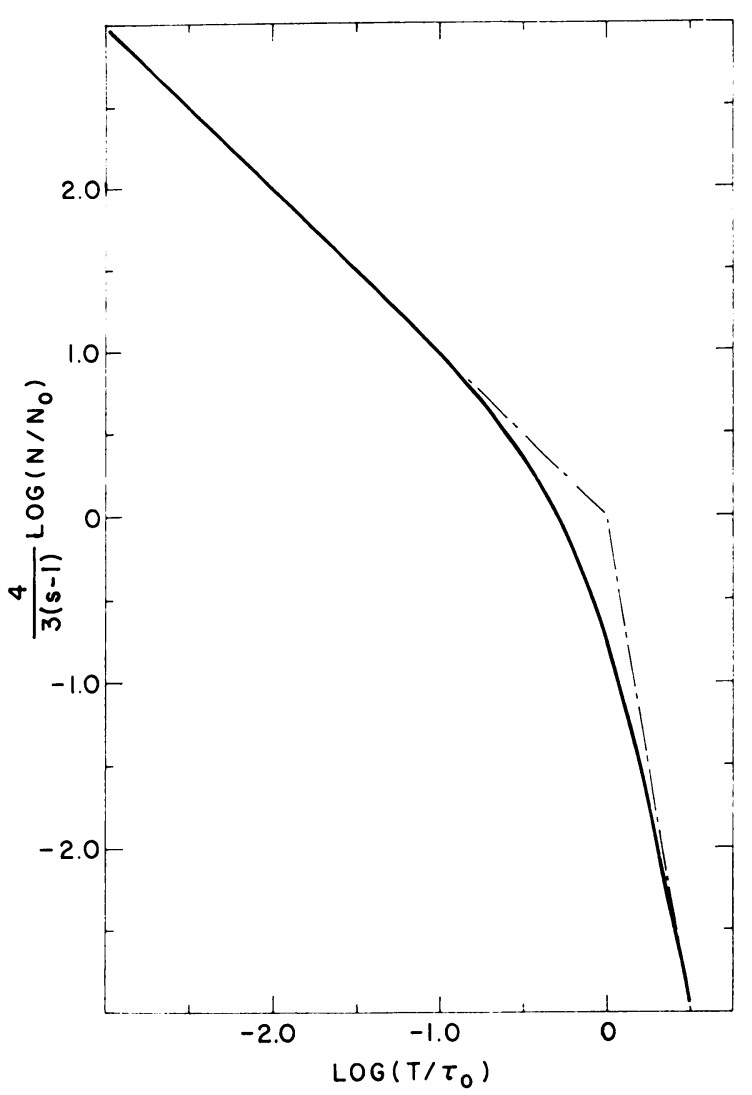

FIG. 2. Theoretical relation between the number of echoes $(N)$ and echo duration $(T)$ down to which count is made. In the linear region at the left, echo duration is controlled by diffusion alone; to the right, duration is controlled by attachment.

from high-velocity meteors such as Perseids and Leonids. Data from the latter shower in 1965 are shown in Figure 5 (from McIntosh and Millman, to be published). The solid curve was calculated according to theory outlined in brief here, and given in more detail in a previous paper (McIntosh, 1966). The equivalent mass scales superimposed on the duration scale indicate in part why a high-velocity shower produces so many long-duration echoes.

We now consider the relation between number of echoes and echo duration for the sporadic background. Suppose that numbers of echoes are measured for a fixed range of durations, say between $1 \mathrm{sec}$ and $10 \mathrm{sec}$, and that the observations are fitted by a relation of the form

$$
\log N=\text { constant }-S \log T .
$$

Since the mean geocentric velocity of that fraction of the meteor population which 


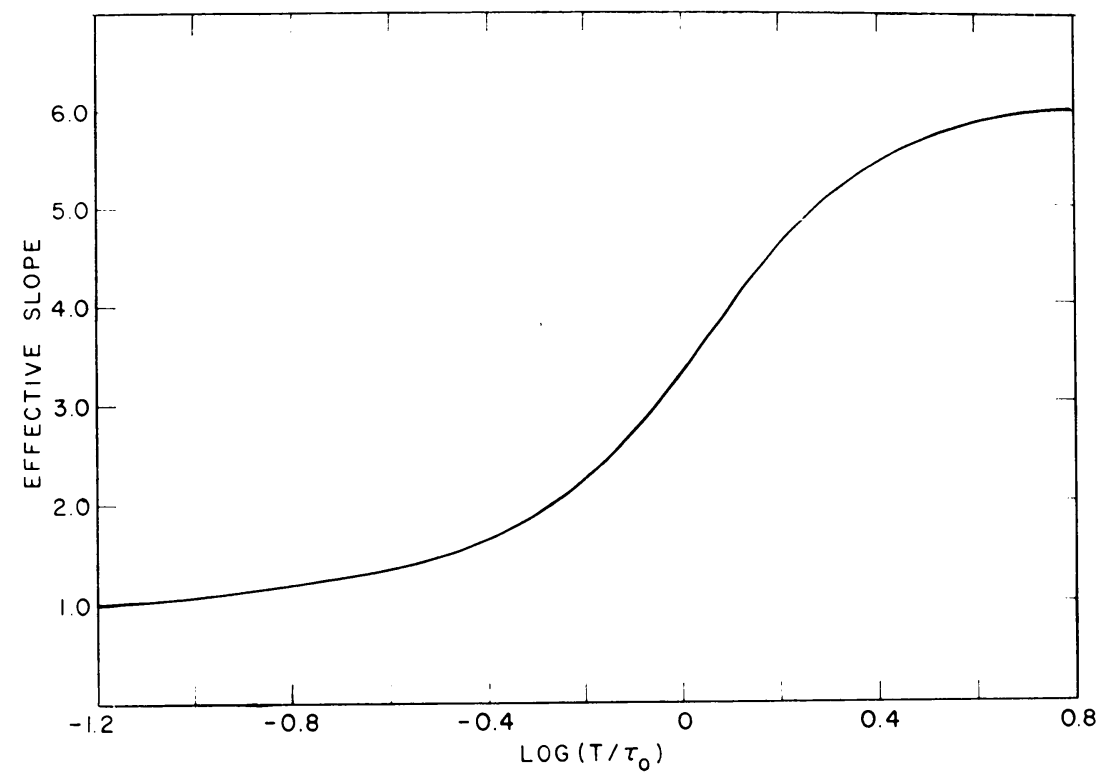

FIG. 3. Plot of the instantaneous slope of the curve of Figure 2 showing the long transition region between diffusion-controlled and attachment-controlled duration.

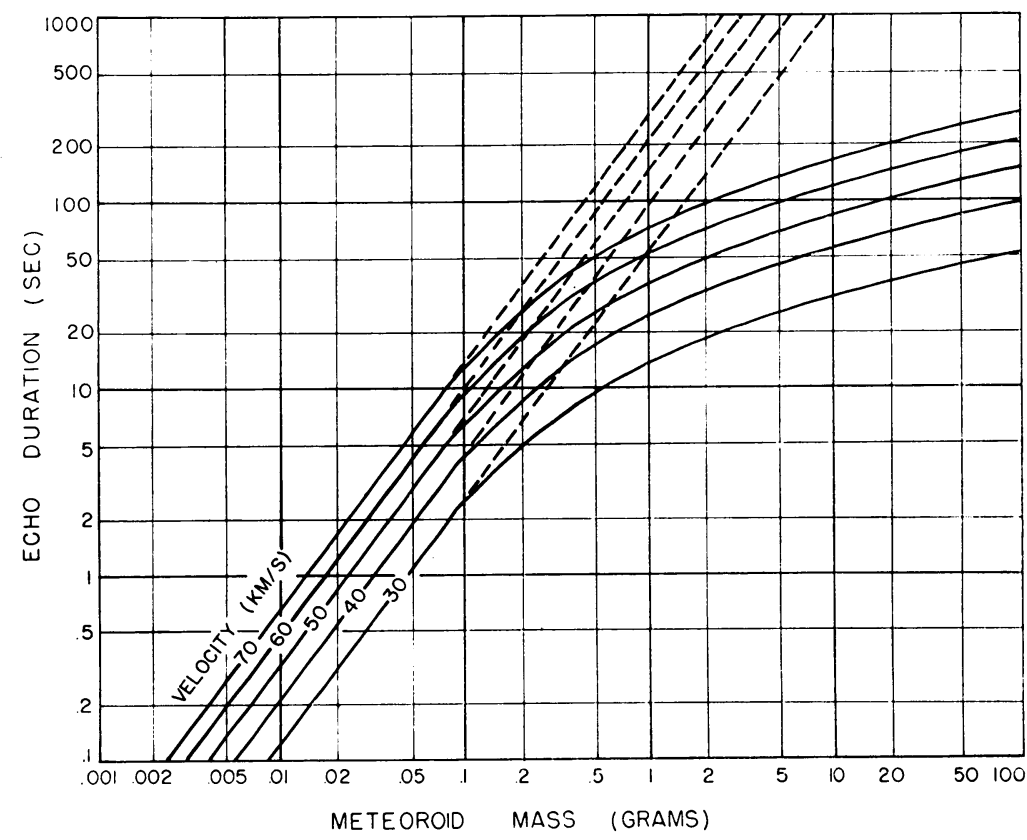

FIG. 4. Approximate echo duration to be expected on a 10-m wavelength radar from a meteoroid of given mass. 


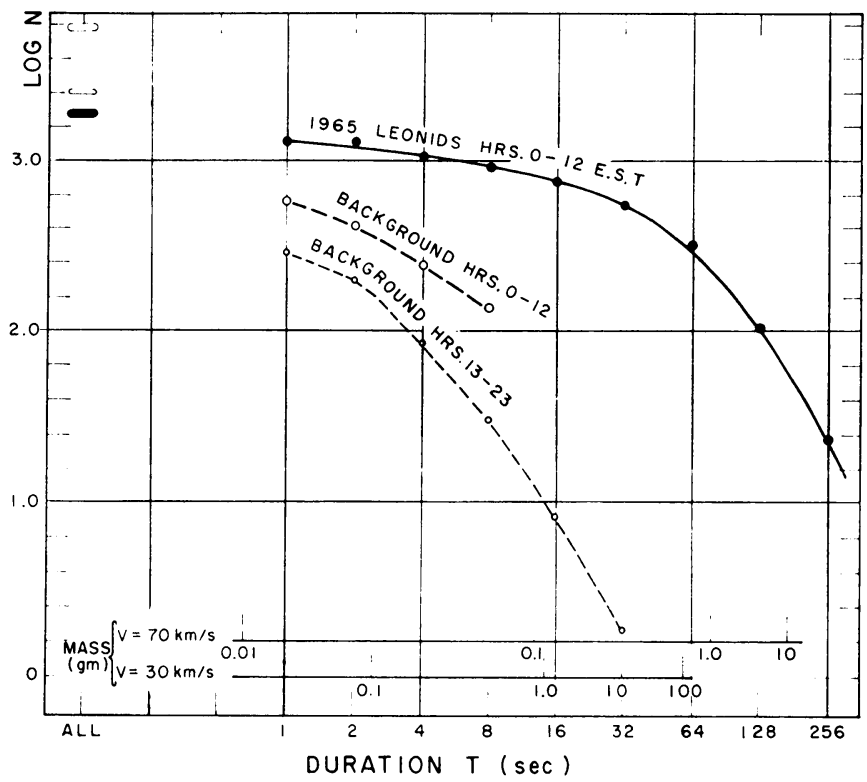

FIG. 5. Observed echo count $(N)$, as a function of duration $(T)$ down to which count was made, during 1965 Leonid shower. The solid curve was calculated using $s=1 \cdot 7, T_{M}=10$ sec and was adjusted vertically to fit the experimental points.

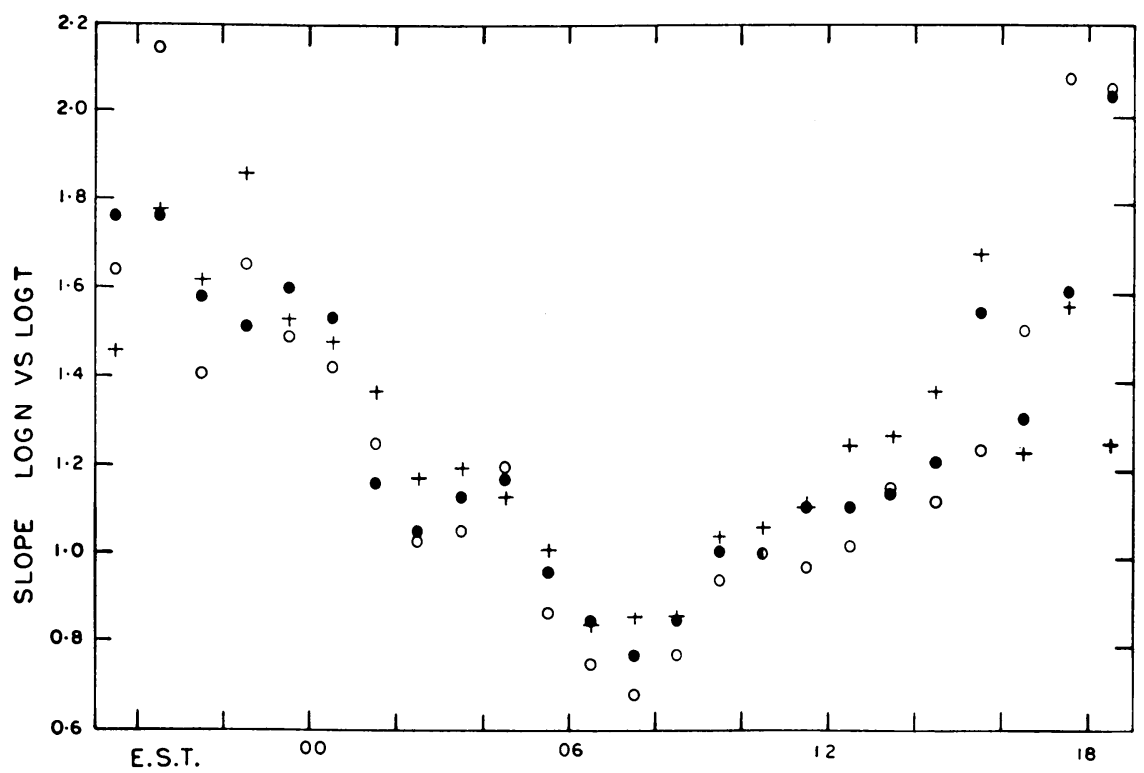

FIG. 6. Apparent diurnal variation of the slope of the observed $\log N$ vs. $\log T$ relation. Data from Springhill (Ottawa) meteor radar, monthly means for March; open circles 1965, filled circles 1966, crosses 1967. 
is observed varies throughout the day and throughout the year, the mean value of $\tau_{0}$ will vary. Thus the position of the $1 \mathrm{sec}$ to $10 \mathrm{sec}$ interval with respect to $\tau_{0}$ will vary. This leads to diurnal and seasonal variations in the $\log N$ vs. $\log T$ slope, $S$, in the chosen duration interval due only to the mean velocity variation. The seasonal variation in slope reported by Millman and McIntosh (1966) can be explained entirely by this effect. A typical diurnal variation is shown in Figure 6. Except near $18^{\mathrm{h}}$ where low number statistics lead to large scatter in the slope values, the variation repeats remarkably well from year to year.

It is worth noting that incautious use of the relation

$$
S=3(s-1) / 4
$$

leads to impossibly high values such as $s=3.4$ for $S=1 \cdot 8$.

If, as proposed here, the seasonal and diurnal changes in $S$ may be due only to the variation in mean velocity, the value of $S$ should be closely associated with the elevation of the Earth's apex, the factor which determines the mean velocity. Qualitatively this is apparent from the seasonal and diurnal variation of $S$, since $S$ is always low when the apex is high, and vice versa. Data from 1965-66 have been examined

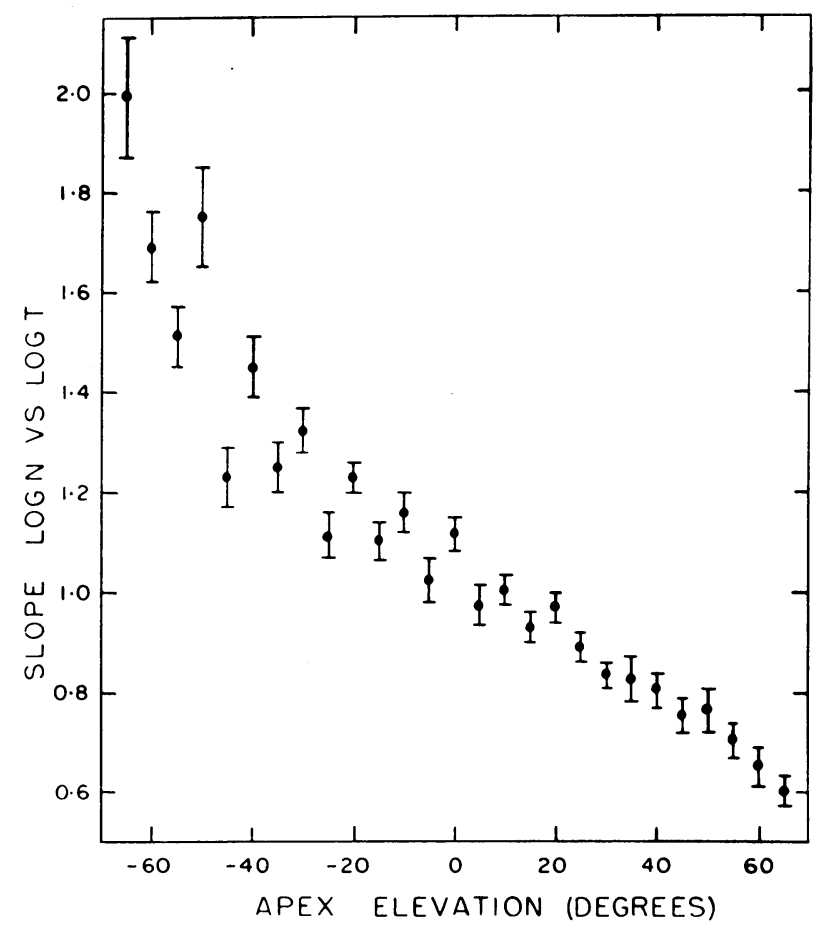

FIG. 7. Mean values of slope $S$ in 5 degree intervals of elevation angle of the Earth's apex. Data from Springhill (Ottawa) meteor radar, monthly means through 1965 and 1966. 
in a more quantitative manner. Slopes for each monthly mean hour during this period have been averaged in $5^{\circ}$ intervals of apex angle. The high correlation between slope $S$ and apex elevation is shown by Figure 7 .

The effect of equipment parameters, in particular antenna beam width, on the statistics of long-duration echoes has been discussed in a previous paper (McIntosh, 1966). If a meteor trail has sufficiently high electron density that it would produce an

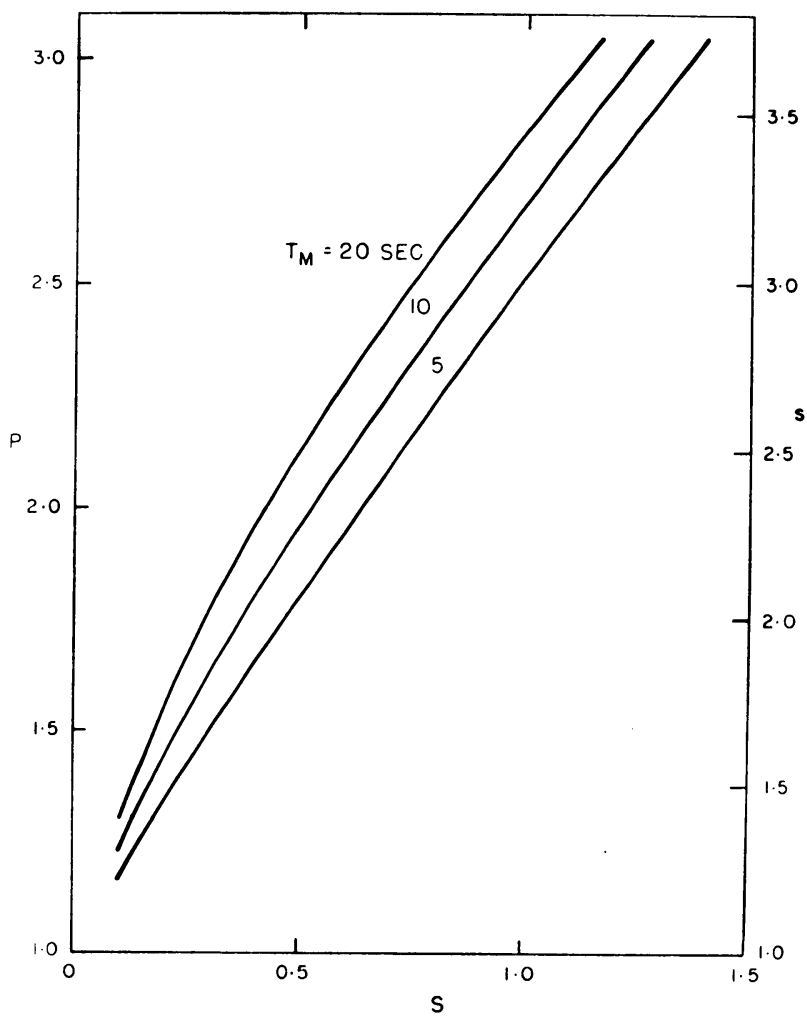

FIG. 8. Theoretical relation between observed slope $S$ and mass exponent $s$ taking into account that long-duration echoes lose all aspect sensitivity after a time $T_{M}$. Applies only if observed echo durations lie entirely in the 'diffusion' region.

echo lasting about $10 \mathrm{sec}$ (at the wavelength of the Ottawa radar) then there is a high probability that it may be seen from any angle, not just perpendicular incidence. This means that the effective collecting area of the antenna system is greater for long-duration echoes ( $>10 \mathrm{sec}$ ) than for short ones. Any antenna system whose beam width is greater than the width of the specular echo 'strip' will show this effect but it is particularly significant for the omnidirectional Ottawa antennas where the enhancement factor is about $\mathbf{4 0}$ times. A correction factor has been deduced (McIntosh, 
1966) for a range of times, $T_{M}$, after which all aspect sensitivity is lost. The curves of Figure 8 show that a value of $s=2 \cdot 3$ which would be expected to yield $S=1 \cdot 0$, in fact gives an observed slope $S=0 \cdot 5$. A direct transformation between $S$ and $s$ can only be used for high-velocity showers such as the Perseids and Leonids. The theoretical curve drawn through the Leonid rates in Figure 5 includes this correction. The minimum value of $S$ reached when the apex elevation is highest (see Figure 7) is $S=0 \cdot 6$. Here it may be that the mean velocity of the meteors is sufficiently high that the transformation of Figure 8 is valid. This indicates an upper limit on the mass exponent $s=2 \cdot 5$.

\section{References}

Greenhow, J.S., Hall, J.E. (1960) Mon. Not. R. astr. Soc., 121, 183.

McIntosh, B. A. (1966) Can. J. Phys., 44, 2729.

McIntosh, B. A. (1967) Can. J. Phys., 45, 3419.

Millman, P. M., McIntosh, B.A. (1966) Can. J. Phys., 44, 1593.

Simek, M., McIntosh, B. A. (1968) in the present volume p. 362.

\section{DISCUSSION}

Levin: What assumptions were made to pass from formula (1) to formula (2)? Was the distribution of reflecting points over the meteor trains (ionized columns) taken into account?

McIntosh: This work was done originally by Kaiser and includes this factor.

Kaiser: The indices in the distribution functions are the same as I obtained in 1953. The theory does take into account the random position of the reflection point on the train.

Belkovič: In our results which have been obtained taking into account the random position of the reflecting points along a meteor train, the slopes of duration distribution are the same. But at the transition region it has another value.

Kaiser: In the expression for $\tau_{0}$, Dr. McIntosh has $\lambda$ to the power $\xi$, in agreement with my own theory which was based on the assumption of two-body attachment. Two-wavelength experiments made at Sheffield, however, give a smaller value than $\frac{2}{5}$, which is consistent with a three-body process. It would therefore be interesting to repeat the calculations for the three-body case.

McIntosh: Evidence from the ionospheric physicists is that attachment rate is proportional to air density squared (three body) only at heights below $93 \mathrm{~km}$. 